\section{ANA-negative Antitopoisomerase I Antibody Is Not Generally Accepted}

\section{To the Editor:}

Recently, Tebo and colleagues suggested that low titer antitopoisomerase I antibody (anti-topo I) including antinuclear antibody (ANA)-negative through multiplex bead assay can be of clinical significance and indicative of the pathogenesis of pulmonary epithelial damage ${ }^{1}$. This suggestion deeply worries us because it may have resulted from a lack of understanding of the methodology.

New sensitive methods have been applied to antiextractable nuclear antigen (anti-ENA) tests; however, their specificity has been low, making their original clinical significance unclear. In similar cases, we have previously pointed out issues related to anti-Sm and antiribosomal $\mathrm{P}^{2,3}$, and a reminder is necessary that the clinical significance of the anti-ENA test (including anti-topo I) was established by the double immunodiffusion method $^{4}$.

In the study by Tebo and colleagues ${ }^{1}$, the median anti-topo I antibody level was significantly higher in patients with systemic sclerosis (SSc) than in patients without it. Regarding the ANA results, all anti-topo I+ patients with SSc were positive, whereas fewer than half $(46.4 \%)$ of anti-topo I+ patients without SSc were positive, which is somewhat predictable. However, these results have some problems.

First, anti-topo I positivity was not screened by ANA test, which is not generally accepted; the authors did not analyze the cause of this discrepancy or consider the possibility of false positives ${ }^{1}$. Second, they did not present the exact number of patients for each disease group; therefore, the basis of their conclusions remains unclear. Using the data revealed by the authors (dots on the graph and the ANA-positive rate in patients without SSc), the number of lung disease cases with low titer anti-topo I is estimated at about 11 patients, 5 of whom are ANA-negative. This number is not only small but raises suspicion about whether the patients are true positives.

The multiplex bead assay method used by the authors to detect anti-topo I antibodies is a sensitive technology that applies both the principles of flow cytometry and sandwich immunoassay with antigen-coated microspheres (beads), allowing multiple assays to be simultaneously performed in a single test ${ }^{5}$. However, there have been repeated warnings of problems caused by this multiplexing reaction: increasing opportunities for nonspecific binding and interference, lot-to-lot variability in bead reactions, and poorly developed quality-control algorithms, among others ${ }^{6}$. In particular, nonspecific binding induced by cross-reactivity due to multiple antigens, substances in the sample, or reagents is the main cause of background fluorescence that results in false positives ${ }^{7}$.

To compensate for this defect, in the real practice of multiplex bead assay measurements, the ANA test should be assigned a screening role of anti-ENA, which is generally recommended. In the Theradiag system used by the authors, the initial dilution of the specimen is higher than the screening dilution of 1:40 that is widely adopted for the ANA test. So it must have been detected on the ANA test no matter how low the concentration of anti-topo I.

In past issues of anti-ENA-positive but ANA-negative findings using indirect immunofluorescence (IF) assays, the main cause was anti-SS-A/Ro owing to possible diffusion or loss of SS-A/Ro antigen during the fixation process of the HEp-2 cell substrate (this is now less common because of the improvement of the cell line and fixatives). On the other hand, for the anti-topo I antibody, the IF pattern could differ somewhat depending on the company's product ${ }^{8}$. However, the positive results were consistent with each other on the ANA test, and to the best of our knowledge, similar problems as those caused by the SS-A/Ro antigen have not been reported. Even though the sensitivity of the ANA test may be affected by subjective factors in the laboratory, ANA-negative anti-topo I is not generally accepted.

Proposing new clinical significance from low anti-topo I results obtained by the multiplex assay method will create confusion, which should be avoided for ANA-negative cases. In Korea, anti-ENA test costs can be charged to the national health insurance system only if the ANA test result is positive. We think such an approach is more reasonable regarding both cost-effectiveness and clinical utility.

LA-HE JEARN ${ }^{\circledR}$, MD, Professor, Department of Laboratory Medicine, College of Medicine, Hanyang University; THINK-YOU KIM ${ }^{-}$, MD, Professor Emeritus, Department of Laboratory Medicine, College of Medicine, Hanyang University, Seoul, Republic of Korea. Address correspondence to Dr. T.Y. Kim, Department of Laboratory Medicine, College of Medicine, Hanyang University, 222-1 Wangsimni-ro, Seongdong-gu, Seoul, 04763, Republic of Korea. E-mail: tykim@hanyang.ac.kr

\section{REFERENCES}

1. Tebo AE, Schmidt RL, Frech TM. Presence of antitopoisomerase I antibody alone may not be sufficient for the diagnosis of systemic sclerosis. J Rheumatol 2019;46:440-2.

2. Jearn LH, Kim TY. Anti-Sm faces a threat to its reigning position as the marker antibody. Rheumatol Int 2011;31:1119-20.

3. Jearn LH, Kim TY. The antiribosomal P antibodies really elicit autoimmune hepatitis? Liver Int 2014;34:165-6.

4. Catoggio LJ, Bernstein RM, Black CM, Hughes GR, Maddison PJ. Serological markers in progressive systemic sclerosis: clinical correlations. Ann Rheum Dis 1983;42:23-7.

5. Fulton RJ, McDade RL, Smith PL, Kienker LJ, Kettman JR Advanced multiplexed analysis with the FlowMetrix system. Clin Chem 1997;43:1749-56.

6. Ellington AA, Kullo IJ, Bailey KR, Klee GG. Antibody-based protein multiplex platforms: technical and operational challenges. Clin Chem 2010;56:186-93.

7. Juncker D, Bergeron S, Laforte V, Li H. Cross-reactivity in antibody microarrays and multiplexed sandwich assays: shedding light on the dark side of multiplexing. Curr Opin Chem Biol 2014;18:29-37.

8. Andrade LEC, Klotz W, Herold M, Conrad K, Rönnelid J, Fritzler $\mathrm{MJ}$, et al. International consensus on antinuclear antibody patterns definition of the AC-29 pattern associated with antibodies to DNA topoisomerase I. Clin Chem Lab Med 2018;56:1783-8.

First Release August 1 2019; J Rheumatol 2019;46:11; doi:10.3899/jrheum.190524 